\title{
NOVEL HOMOZYGOUS VARIANT OF TBC1 DOMAIN FAMILY MEMBER 8 GENE IN FOUR LIBYAN SIBLINGS WITH AUTISTIC SPECTRUM DISORDER AND INTELLECTUAL DISABILITY WITHOUT EPILEPSY
}

\author{
ADEL ZEGLAM ${ }^{1}$, SUAD ALHMADI ${ }^{2}$ \\ ${ }^{1}$ Department of Pediatrics, Faculty of Medicine, Tripoli University Hospital, Tripoli, Libya. ${ }^{2}$ Department of Paediatrics, Al-Khadra General \\ Hospital, Tripoli, Libya. Email: adelzeglam@gmail.com
}

Received: 11 February 2021, Revised and Accepted: 07 May 2021

\section{ABSTRACT}

Objective: Recent progress in genetic analysis and investigations have enabled researchers to identify potential genetic changes that may play a role in ASD. The number of genes connected with autism is growing. Whole exome sequencing(WES) identified the homozygous TBC1D8 variant. Aim to report for the first time a TBC1D8 missense variant (c.1883G>A, p. (Arg628Gln) in 4 Libyan children (3 homozygous,1 heterozygous) with severe neurodevelopmental phenotypes ASD and intellectual disability ID. Based on the data of HGMD and ClinVar, variants in only a few autosomal recessive intellectual disability ARID genes seem to be reported frequently.

Method: Molecular genetic analysis of (WES) was carried out on blood samples from these children. The outcome of the genetic investigations was interpreted within the context of clinical finding, family history, and suspected mode of inheritance.

Results: The number of genes associated with autism is increasing. WES identified the TBC1D8 variant. According to the longest isoform (NM_001102426.1),the nomenclature of this variant is c.1883G>A, p. (Arg628Gln) in TBC1D8 which leads to an amino acid exchange. This variant has not previously reported or described in the literature (PubMed, HGMD).

Conclusion: we have provided evidence for a connection between TBC1D8 variant and ASD and ID; however, this evidence should be considered preliminary in the context of a single case report and such findings need to be replicated to gain insight in order to determine if ASD and ID are a characteristic of this variant.

Keywords: TBC1D8, Autism, Libya, Epilepsy.

(C) 2021 The Authors. Published by Innovare Academic Sciences Pvt Ltd. This is an open access article under the CC BY license (http://creativecommons. org/licenses/by/4.0/) DOI: http://dx.doi.org/10.22159/ijms.2021v9i6.41029. Journal homepage: https://innovareacademics.in/journals/index.php/ijms

\section{INTRODUCTION}

TBC1 Domain Family Member 8 (TBC1D8) is a Protein Coding gene. Cell cycle is one of the gene-associated pathways. Annotations linked to gene ontology (GO) include calcium ion binding and activation of GTPase. An important paralog of this gene is TBC1D8B (HGNC: 17791) (GCID: GC02M101007).The chromosome location is 2q11.2. No more than a few of the novel ID genes seem to share to molecular pathways and networks that have been earlier involved in dominant and X-linked forms of ID, such as pre-and post-synaptic signaling, transcription regulation, and epigenetic mechanisms [1-3]. The only exemption is UBE2]2 encoding a ubiquitin-conjugating enzyme, which symbolizes an evolving mechanism for controlling synapse function by post-translational alteration through the ubiquitin pathway at the postsynaptic membrane [4]. Proteolysis by the ubiquitin proteosome pathway is recognized as a major molecular pathway leading to several neurodevelopmental and neurodegenerative diseases [5]. Previously identified gene networks that are commonly disrupted in IDand other neurodevelopmental disorders are composed of genes that are highly dosage-sensitive. Therefore, these pathways might indicate other neurobiological activities than those that are affected by recessive mutations [6]. In this article we report for the $1^{\text {st }}$ time a homozygous TBC1D8 missense variant (c.1883G $>$ A, p. (Arg628Gln) in 3 Libyan children with severe neurodevelopmental phenotypes (ASD) and (ID). Surprisingly, the fourth sibling carries the TBC1D8 variant in heterozygous state. According to the American Association on Intellectual and Developmental Disabilities, Intellectual disability is at present is regarded as "a disability associated with significant restrictions both in intellectual functioning and in adaptive behavior as expressed in conceptual, social, and practical adaptive skills. This disability originates before age 18" [7]. Moreover, newly published DSM-5 uses the term "intellectual developmental disorder" as the alike term for "intellectual disability" because forthcoming ICD-11 will probably use the term "intellectual developmental disorder" to show involvement of compromised brain functioning earlyin life [8]. Autistic spectrum disorder (ASD) comprises a group of neurobiological disorders that are characterized by deficits in social interaction and communication and by abnormalities in behaviors, interests, and activities [8]. ASD has a complex genetic etiology, and monogenic disorders associated with the high penetrance of ASD are observed in less than 20\% of ASD cases. Well-known single-gene disorders associated with ASD are Fragile X syndrome, Rettsyndrome, MECP2 duplication, Angelman syndrome, and Tuberous sclerosis [9].

NewUSA government survey (2014) of parents suggests that 1 in 45 children aged 3 through 17 had been diagnosed with ASD. This is remarkably higher than the formal government approximate of 1 in 68 American children with autism, as stated by National Center for Health Statistics [10]. One prospective hospital-based study conducted on all children referred to the neurodevelopment clinic at Al-Khadra Teaching Hospital, Tripoli, Libya between 2005 and 2009 for ASD evaluation showed a prevalence of one in 300 [11]; yet again in the 2011 study 200 children were referred for ASD evaluation because of their behavioral difficulties and speech and language disorders. ASD was diagnosed in $83 \%$ of cases ( 166 children) which gives a prevalence of 10:1000 [12,13]. The prevalence of the problem in Libya is probably higher or similar to that seen in the USA and UK. 


\section{METHODS}

These four Libyan children were born to non-blood-related parents at term after uneventful pregnancy. Parents provided informed signed consent for genetic studies on blood samples from their children. The outcome of the genetic investigations was interpreted in the context of clinical finding, family history, and suspected mode of inheritance.

\section{Children}

Child 1 (girl) was the first sib. She is now 12 year old. Delayed developmental milestones including speech and language were noted since early infancy. At the age of 1 year: she was noted to have poor eye to eye contact; and the diagnosis of (ASD) and (ID) was made at 2 years of age. Child 2 (girl) is the second sib who is now 10 years old. Developmental delay was noted since early infancy. At the age of 20 months, her eye-to-eye contact was still absent. No seizures were reported. Speech was absent; and the diagnosis of (ASD) and (ID) were made at 4 years of age. Child 3 (boy) is the third sib who is now 9 years old. Developmental delay was also noted since early infancy. At the age of 18 months, his eye contact was absent. No seizures were reported. Speech was absent; and the diagnosis of (ASD) and (ID) was made at 5 years of age. Child 4 (girl) is the fourth sib who is now 7 years old. Developmental delay was noted since early infancy. At the age of 24 months, she was noted to have inconsistent imitation skills. Eye contact was absent. No seizures were reported. Speech was absent; and the diagnosis of (ASD) and (ID) was made at 5 years of age.

\section{Genetic studies}

Molecular genetic analysis of whole-exome sequencing (WES) was carried out at Bioscientia Institute for Medical Diagnostics Gmbh, Center for Human Genetics, Ingelheim, Germany. More than 20,000 genes of the patients DNA were enriched and sequenced.Filtering of the exome data targeted recessive, X-linked, and dominantly inherited disease. Genomic DNA was fragmented and the exons of the known gene in the human genome, as well as corresponding exon-intron boundaries, were enriched using Roche Nimble Gen capture technology(SeqCap MedExome Library)amplified and sequenced simultaneously by illumina technology(next-generation sequencing NGS) using an illumina system. The target regions were sequenced with average coverage of 164 fold. For about $97 \%$ of the regions of interest a 15 -fold coverage, for about $95 \%$ a 20 -fold coverage was obtained. NGS data were aligned to the hg19 genome assembly. Variant calling and annotation were performed by an in-house developed bioinformatics pipeline identified single-nucleotide variants and indels were filtered against external and internal database focusing on rare variants with minor allele frequency in gnomAD of $1 \%$ or less and removing known artifact and variants in regions with highly homologous regions. Classification of variants was conducted based on ACMG guidelines considering database entries (incl.HGMD), bioinformatics prediction tools, and literature status [14]. A change of pathogenicity classifications over time cannot be excluded from the study. Variants annotated as common polymorphisms in databases or literature or that were classified as (likely) benign were neglected. Putatively pathogenic differences between the wild type sequence (human reference genome according to (UCSC Genome Browser: hg19, GRCh37) and the patients sequence mentioned and interpreted in this report were assessed using an in-house established quality score. Variants not passing the quality threshold were verified using polymerase chain reaction amplification followed by conventional Sanger sequencing. Sample identity was ensured by internal quality management procedures. The laboratory Quality management is also recognized and accredited by the Central Office of the Federal States for Health Protection for Drugs and Medical Devices (ZLG), German accreditation body (DAkkS) and College of American Pathologists. The recorded data in paper and/or in electronic form are stored in accordance with the legal requirements and their use and/or publication should be in pseudonymized form for scientific purposes. Confidentiality is maintained. Any sample material that remaining at the end of the analysis is transferred, in accordance with $\S 950$ BGBI (Germany's official gazette of federal law).

\section{DIAGNOSIS AND MANAGEMENT}

ASD and ID were established as follows: First, according to history, parent interview and neurodevelopmental symptoms, including cognition, motor performance, hearing, speech, and vision; second, through assessment of intelligence quotient (IQ) using a standardized tests (IQ below 70); third, according to limitations in environment adaptation, including self-care and skills of communication $[15,16]$. Neurodevelopmental assessment with the help of the vineland established adaptive behavior scale (VABS), DSM5, Modified checklist of autism in children(M-CHAT), and Conner's Autism Rating Scale revealed that the children were suffering from autism along with severe ID with an IQ score of about 25-30 [17-19].

Auditory brainstem response showed normal hearing.EEG revealed no abnormal epileptic activities; and MRI brain showed normal brain structure with no evidence of encephalopathy, degenerative changes, or atrophy. Their complete blood pictures, urea and electrolytes, liver and thyroid function tests, and creatinine kinase were normal. The metabolic screen including serum bicarbonate, arterial blood gases, Vitamin D and B12 were normal. Molecular genetic analysis of WES identified the homozygous missense variant in the TBC1D8 gene in the entire children (Table 1).

\section{RESULTS}

The number of genes related with autism is increasing [20]. WES identified the homozygous TBC1D8 variant. The following is NCBI Reference Sequence: LOCUS: NM_001102426 (4226 bp - mRNA - linear - PRI 07-MAY-2020) DEFINITION: Homo sapiens TBC1D8, transcript variant 2, mRNA. ACCESSION: NM_001102426 VERSION: NM_001102426.2 KEYWORDS: RefSeq. SOURCE: Homo sapiens (human) The nomenclature of this variant is c.1883G $>A$, p. (Arg628Gln) in TBC1D8 which leads to an amino acid exchange. Eight out 10 bioinformatic in silico programs predict a pathogenic effect for this variant. In silico analysis of the variant showed that 17 out of 30 tools predicted a possible pathogenicity (Prediction [17/30] = Functional [11/22] + Conservation [6/8]). While many deleteriousness prediction methods have been developed, their prediction results are sometimes not in agreement with each other. The predictive performance of 18 current deleteriousness-scoring methods was comprehensively evaluated, including 11 function prediction scores (PolyPhen-2, SIFT, MutationTaster, Mutation Assessor, FATHMM, LRT, PANTHER, PhD-SNP, SNAP, SNPs\&GO and MutPred), 3 conservation scores (GERP++, SiPhy, and PhyloP) and 4 ensemble scores (CADD, PON-P, KGGSeq, and CONDEL). It has been found that FATHMM and KGGSeq had the maximum discriminative power among independent scores and ensemble scores, respectively [21]. This variant has not formerly been reported or described in the literature (PubMed, HGMD). The variant is found in $0.0014 \%$ of the whole population(4 heterozygous, 0 homozygous; $\mathrm{X}$ hemizygous; GnomAD). (Riazuddin and his colleagues reported a c.1501C $>$ T, p. (Leu501Phe) variant of TBC1D8 in a Punjabi consanguineous family (PKMR66) among the other 30 novel candidate genes for autosomal recessive intellectual disability (ARID) with neither epilepsy nor ASD (Table 2) [6].

\section{DISCUSSION}

A global prevalence of (ASD) was estimated on review of epidemiological studies and has been found to be increasing [22]. Improvements in genetic analysis have offered the ability to recognize potential genetic variations that may contribute to ASD. Remarkably, based on the data of HGMD and ClinVar, variants in only a few ARID genes appear to be reported frequently. The large ARID studies that were executed on over 100 families none of them showed any remarkably prevalent gene in ARID. It isextremelysignificant totake note that the final conclusion from molecular genetic analysis of WES must not end with laboratory classification or grouping of a variant or variants. For example, the classification of a variant of unknown significance (VUS) from a laboratory should be further evaluated in the clinical setting. With 
Innovare Journal of Medical Science, Vol 9, Issue 6, 2021, 1-4

Table 1: Molecular genetic analysis of whole-exome sequencing

\begin{tabular}{llllll}
\hline Gene (Isoform) & $\begin{array}{l}\text { OMIM-P } \\
\text { (Mode of } \\
\text { inheritance) }\end{array}$ & Variant & Zygosity & $\begin{array}{l}\text { Minor allele frequency } \\
\text { gnomAD (\%) }\end{array}$ & $\begin{array}{l}\text { Literature } \\
\text { (PIMD) }\end{array}$ \\
\hline TBC1D8 (NM_001102426.1 & - & $\begin{array}{l}\text { c.1883G>A } \\
\text { p.(Arg628Gin) } \\
\text { chr2:101648738 }\end{array}$ & Hom. & 0.0014 & - \\
\hline
\end{tabular}

Table 2: Homozygous DNA variants in a single gene in a Punjabi ID consanguineous family

\begin{tabular}{|c|c|c|c|c|c|c|c|c|}
\hline Family & Ethnicity & Gene & Position & Transcript & $\begin{array}{l}\text { cDNA } \\
\text { mutation }\end{array}$ & $\begin{array}{l}\text { Protein } \\
\text { change }\end{array}$ & variation & $\begin{array}{l}\text { Protein } \\
\text { function }\end{array}$ \\
\hline PKMR66 & Punjabi & TBC1D8 & Chr2:101652537G>A & NM_001102426.1 & c.1501C4T & p.(Leu501Phe) & Missense & $\begin{array}{l}\text { G-protein } \\
\text { modulator } \\
31.099 .37\end{array}$ \\
\hline
\end{tabular}

increased and betterunderstanding over time of phenotypes linked to different genetic disorders, exome reanalysis may change the clinical interpretation of a VUS [23]. Homozygous mouse models (Tbc1d8 em1 [IMPC] J/Tbc1d8 em1 [IMPC] J) showed abnormal behavior, cataract and male infertility(MGI Reference; 1927225 ID = J:188991) [24,25]. Numerous existing techniques for pathway examination rely upon prevailing databases. The information utilized is not in every case totally clarified. Many genes interactions in databases are somewhat theoretical as they depend on scientific facts and are pulled from a particular cell type or illness. In addition, most authoritative pathways are manufactured utilizing the information got from an inadequate number of analyses with limited cell models [26]. This series of four Libyan siblings with ASD and ID; sum-ups novel presentations of a variant in protein coding genes TBC1D8 (c.1883G>A, p. (Arg628Gln). The four siblings had what appeared to be similar presentation of ASD at initial assessment and have continued through the similar clinical course, progressed in the same way but regrettably they have been making slow and disappointing progress despite very good educational input. Generally speaking, these four cases provide novel presentations of ASD and ID and highlight the importance ofWES investigations. To our knowledge, the variant has not been described in the literatures so far. The variant is found in $0.0014 \%$ of the overall population (4 heterozygous, 0 homozygous; $\mathrm{X}$ hemizygous,gnomAD). Taken together, it is clear that the detected homozygous TBC1D8 variant may contribute to the phenotype of these siblings.

\section{CONCLUSION}

In this article, we have provided evidence for a plausible connection between TBC1D8 variant and ASD and ID; however, this evidence should be considered preliminary within the framework of a single case report of four siblings and such findings need to be replicated in order to determine if ASD and ID are a characteristic of this variant; indeed, we report these findings to encourage other clinicians and professionals to look for such variants when dealing with children who suffers from ASD and ID. Theawareness, understanding and knowledge about the causes of human genetic disorders constantly improve due to the continuous identification of novel disease genes.

\section{ACKNOWLEDGMENT}

We extend great thanks and appreciation to all the parents for their support and to all those involved in running this study. The authors would like to thank Bioscientia Healthcare Group based in Ingelheim near Frankfurt, Germany, for providing of laboratory services and all the genetic investigations.

\section{AUTHOR CONTRIBUTIONS STATEMENT}

AMZ made a substantial contributed to the conception and design of the work. AMZ drafted the work or revised the article criticallyforimportant intellectual content. AMZ provided approval for publication of the content. SA supervised the collection and labeling of blood samples. SA word processed the daft and the main article. AMZ and SA Agreed to beaccountable for all aspects of the workin ensuringthat questions associated to the accuracy or integrity of any part of the work are appropriately investigated and resolved.

\section{CONFLICT OF INTEREST}

We have no conflicts of interest to disclose. All procedures performed were in accordance with the ethical standards of the hospital. Informed consent was obtained from children 'parent.

\section{FUNDING SOURCE}

No funding was secured for this study.

\section{FINANCIAL DISCLOSURE}

The authors have no financial relationships applicable to this article to disclose.

\section{REFERENCES}

1. van Bokhoven H. Genetic and epigenetic networks in intellectual disabilities. Annu Rev Genet 2011;45:81-104.

2. McCarthy S, Gillis J, Kramer M, Lihm J, Yoon S, Berstein Y, et al. De novo mutations in schizophrenia implicate chromatin remodeling and support a genetic overlap with autism and intellectual disability. Mol Psychiatry 2014;19:652-8.

3. de Rubeis S, He X, Goldberg A, Poultney CS, Samocha K, Cicek AE, et al. Synaptic, transcriptional and chromatin genes disrupted in autism. Nature 2014;515:209-15.

4. Mabb AM, Ehlers MD. Ubiquitination in postsynaptic function and plasticity. Annu Rev Cell Dev Biol 2010;26:179-210.

5. Hegde AN, Upadhya SC. Role of ubiquitin-proteasome-mediated proteolysis in nervous system disease. Biochim Biophys Acta 2011;1809:128-40.

6. Riazuddin S, Hussain M, Razzaq A, Iqbal Z, Shahzad M, Polla DL, et al. Exome sequencing of Pakistani consanguineous families identifies 30 novel candidate genes for recessive intellectual disability. Mol Psychiatry 2017;22:1604-14.

7. AAIDD (American Association on Intellectual Developmental Disabilities). Intellectual Disability: Definition, Classification, and Systems of Supports. Washington, DC: AAIDD; 2010.

8. APA (American Psychiatric Association). Diagnostic and Statistical Manual of Mental Disorders. $5^{\text {th }}$ ed. Washington, DC: APA; 2013.

9. Sztainberg Y, Zoghbi H. Lessons learned from studying syndromic autism spectrum disorders. Nat Neurosci 2016;19:1408-17.

10. CDC, National Center for Health Statistics. Estimated Prevalence of Autism and Other Developmental Disabilities Following Questionnaire Changes in the 2014 National Health Interview Survey, No. 87; 2015. Available from: http://www.cdc.gov/nchs/products/nhsr.htm

11. Zeglam AM, Maouna A. Is there a need for a focused health care service for children with autistic spectrum disorders? A keyhole look at 
this problem in Tripoli, Libya. Autism 2012;16:337-9.

12. Zeglam AM, Maouna AJ. Prevalence of autistic spectrum disorders in Tripoli, Libya: The need for more research and planned services. East Mediterr Health J 2012;18:184-8.

13. Zeglam AM, Al-Bloushi H. Autism today in Libya: Is it a tip of an iceberg? A comparative study. World Fam Med J 2012;10:34-8.

14. Richards S, Aziz N, Bale S, Bick D, Das S, Gastier-Foster J, et al. Standards and guidelines for the interpretation of sequence variants: A joint consensus recommendation of the American college of medical genetics and genomics and the association for molecular pathology. Genet Med. 2015;17:405-24.

15. Emery AEH, Rimoin DL, editors. Emery and Rimoin's Principles and Practice of Medical Genetics. London, United Kingdom: Churchill Livingstone, Elsevier; 2007.

16. Moeschler JB, Shevell M, American Academy of Pediatrics Committee on Genetics. Clinical genetic evaluation of the child with mental retardation or developmental delays. Pediatrics 2006;117:2304-16.

17. Sparrow SS, Cicchetti DV, Saulnier CA. Vineland-3: Vineland Adaptive Behavior Scales. $3^{\text {rd }}$ ed. Minneapolis, MN, USA: Pearson Assessments 2016 .

18. Autism Speaks. Modified Checklist for Autism in Toddlers, Revised (M-CHAT-R); 2016. Available from: http://www.autismspeaks.org/ what-autism/diagnosis/screen-your-child
19. Conners CK. Conners' Rating Scales-Revised Technical Manual. North Tonawanda. New York: Multi Health Systems; 1997.

20. Satterstrom FK, Kosmicki JA, Wang J, Breen MS, Rubis SD Collins $\mathrm{R}$, et al. Large-scale exome sequencing study implicates both developmental and functional changes in the neurobiology of autism. Cell 2020;180:568-84.e23.

21. Dong C, Wei P, Jian X, Gibb R, Boerwinkle E, Wang K, et al. Comparison and integration of deleteriousness prediction methods for nonsynonymous SNVs in whole exome sequencing studies. Hum Mol Genet 2015;24:2125-37.

22. Baxter AJ, Brugha TS, Erskine HE, Scheurer RW, Vos T, Scott JG. The epidemiology and global burden of autism spectrum disorders. Psychol Med 2015;45:601-13.

23. Wright CF, Fitzpatrick DR, Firth HV. Paediatric genomics: Diagnosing rare disease in children. Nat Rev Genet 2018;19:253-68.

24. Bult CJ, Blake JA, Smith CL, Kadin JA, Richardson JE, The Mouse Genome Database Group. Mouse genome database (MGD) 2019 Nucleic Acids Res 2019;47:D801-6.

25. Smith CM, Hayamizu TF, Finger JH, Bello SM, McCright IJ, Xu J, et al. The mouse gene expression database (GXD): 2019 Update. Nucleic Acids Res 2019;47:D774-9.

26. Epi25 Collaborative. Ultra-rare genetic variation in the epilepsies: A whole-exome sequencing study of 17, 606 individuals. Am J Hum Genet 2019;105:267-82. 